\title{
Práticas Parentais: Conjugalidade, Depressão Materna, Comportamento das Crianças e Variáveis Demográficas
}

\author{
Alessandra Turini Bolsoni-Silva ${ }^{1}$ \\ Sonia Regina Loureiro ${ }^{2}$ \\ ${ }^{1}$ Universidadae Estadual Paulista "Tulio de Mesquita Filho", Bauru, SP \\ ${ }^{2}$ Universidade de São Paulo, Ribeirão Preto, SP
}

\begin{abstract}
Resumo
As interfaces das práticas educativas maternas e problemas de comportamento são reconhecidas, contudo, carecem de estudos que abordem variáveis contextuais e pessoais específicas que podem interferir no uso de práticas positivas e negativas. Objetiva-se comparar as práticas parentais relatadas pelas mães de crianças pré-escolares e escolares considerando variáveis: demográficas, práticas educativas, relacionamento conjugal, depressão materna, problemas de comportamento e habilidades sociais. Foram participantes 151 mães biológicas e suas crianças. Estas informaram sobre práticas parentais, comportamentos dos filhos, depressão materna e variáveis demográficas. Com significância estatística identificou-se que: as habilidades sociais infantis, problemas de comportamento, relacionamento conjugal, escolaridade e renda familiar diferenciaram os grupos no que refere ao uso de práticas educativas; práticas positivas foram associadas às habilidades sociais infantis, e as negativas, aos problemas de comportamento; o uso das práticas positivas foi influenciado, para os escolares, pela depressão, renda familiar e escolaridade materna e, para os pré-escolares, pela escolaridade materna.
\end{abstract}

Palavras-chaves: práticas educativas, famílias, depressão, habilidades sociais, problemas de comportamento, variáveis demográficas

\section{Parental Practices: Conjugality, Maternal Depression, Children's Behavior and Demographic Variables}

\begin{abstract}
Interfaces between maternal educational practices and behavior problems are recognized. However, there are few studies that approach context variables and specific people that can interfere using both positive and negative practices. The objective of this study was to compare parental practices reported by mothers of pre-school and elementary school children, considering variables such as demographic data, educational practices, marital relationship, maternal depression, behavior problems and social skills. Participants included a total of 151 biological mothers and their children, who reported on parental practices, children behavior, maternal depression and demographic variables. With statistical significance, it was identified that: children's social skills, behavior problems, marital relationship, education level and familiar income distinguished the groups in relation to the use of educational practices; positive practices were associated to children's social skills, whereas negative practices were linked to behavior problems; For elementary school children, the use of positive practices was influenced by depression, familiar income and maternal schooling, and for pre-school children, maternal schooling was the influencing factor.

Keywords: educative practices; families; depression; social skills; behavior problems; demographic variables
\end{abstract}

\section{Prácticas Parentales: Conjugalidad, Depresión Materna, Comportamiento de los Niños y Variables Demográficas}

\section{Resumen}

Las interfaces de las prácticas educativas maternas y los problemas de comportamiento, son reconocidos, sin embargo, carecen de estudios que aborden variables contextuales y personales específicas que puedan interferir en el uso de prácticas positivas y negativas. Se pretende comparar las prácticas parentales relatadas por las madres de niños preescolares y escolares considerando las siguientes variables: demográficas, prácticas educativas, relaciones conyugales, depresión materna, problemas de comportamiento y habilidades sociales. Participaron 151 madres biológicas y los hijos. Estas informaron sobre prácticas parentales, comportamientos de sus hijos, depresión materna y variables demográficas. Con alto significado estadístico se identificó que: las habilidades sociales infantiles, problemas de comportamiento, relacionamiento conyugal, escolaridad y renta familiar diferenciaron a los grupos en lo que se refiere al uso de prácticas educativas; las prácticas positivas se asociaron a las habilidades sociales infantiles y las negativas, a los problemas de comportamiento; el uso de las prácticas positivas fue influenciado en los escolares, por la depresión, renta familiar y escolaridad materna; y en los preescolares, por la escolaridad materna.

Palabras-clave: prácticas educativas; familias; depresión; habilidades sociales; problemas de comportamiento; variables demográficas

A interação social entre pais e crianças, segundo Bolsoni-Silva, Loureiro e Marturano (2016), pode ser descrita em três eixos, a saber, comunicação, afeto e estabelecimento de limites os quais se expressam por meio de práticas parentais positivas/habilidosas ou não habilidosas, por meio de práticas negativas. Segundo Gomide (2006), as práticas positivas podem ser caracterizadas por monitoria positiva e ensino do 
comportamento moral, enquanto que as negativas incluem negligência, monitoria negativa, ausência de afeto/atenção, disciplina relaxada, punição física, abuso físico e punição inconsistente.

As práticas positivas parecem prevenir o surgimento de problemas de comportamento e promover habilidades sociais (Berry \& O'Connor, 2010; Bolsoni-Silva, Loureiro, \& Marturano, 2016; Borden et al., 2014; Leme \& Bolsoni-Silva, 2010; Marin, Piccinini, Gonçalvez, \& Tudge, 2012), especialmente quando há consistência no reforçamento das habilidades sociais infantis (Leme \& Bolsoni-Silva, 2010). Por outro lado, as práticas negativas, especialmente as punições e ameaças, parecem aumentar a probabilidade de problemas de comportamentos externalizantes (Price, Chiapa, \& Walsh, 2013; Trepat, Granero, \& Ezpeleta, 2014) e internalizantes (Xing \& Wang, 2013), quando utilizadas de maneira inconsistente (Leme \& Bolsoni-Silva, 2010). As práticas parentais se relacionam a diferentes respostas comportamentais das crianças, podendo exercer diferentes funções no desenvolvimento socioemocional, favorecendo-o ou prejudicando-o (Alvarenga, Weber, \& Bolsoni-Silva, 2016).

Considera-se que as interações entre pais e filhos constituem-se em um cenário complexo para o qual concorre um amplo conjunto de variáveis, que envolvem condições sociodemográficas, variáveis do contexto familiar e condições pessoais dos pais, as quais serão abordadas a seguir quanto à especificidade tratada neste estudo.

Variáveis demográficas das crianças, como sexo e escolaridade, também parecem influenciar os comportamentos infantis. Ainda que não haja consenso nos estudos da área (Wichstrøm, Belsky, \& Berg-Nielse, 2013), a maioria deles indica uma maior prevalência de problemas comportamentais externalizantes em meninos (Borden et al., 2014; Cosentino-Rocha \& Linhares, 2013; Landale, Lanza, Hillemeier, \& Oropesa, 2013), e internalizantes entre as meninas (Cosentino-Rocha \& Linhares, 2013), embora essas também sejam avaliadas como tendo mais habilidades sociais (Leman \& Bjornberg, 2010). Por outro lado, Martín, Granero e Ezpeleta (2014) não identificaram associação entre sexo e problemas comportamentais. Achados quanto à influência da escolaridade no repertório infantil também não são consensuais, mas pode-se dizer que as crianças escolares parecem ser mais habilidosas que as pré-escolares (Berry \& O'Connor, 2010). Entretanto, Bolsoni-Silva et al. (2016) não identificaram diferenças quanto às práticas educativas a partir da escolaridade infantil, tendo verificado mais escores de habilidades sociais e de problemas de comportamento nos pré-escolares que nos escolares.

Também, com relação às variáveis sociodemográficas, é relevante destacar a influência para as práticas parentais e comportamentos das crianças da escolaridade dos pais e da renda familiar. Estudos indicaram que os pais que têm mais escolaridade se preocupam mais com os comportamentos dos filhos (Algarvio, Isabel, \& Maroco, 2013) e ainda Buehler e Gerard (2013) identificaram que o baixo nível educacional dos pais e a baixa renda foram preditores de problemas de ajustamento.

Dentre as variáveis do contexto familiar destacar-se-á a configuração familiar, enquanto famílias compostas pela presença dos pais se contrapondo a famílias monoparentais, que contam com um cuidador único. Estudos que comparam composições familiares diversas sinalizam que a convivência com ambos os pais é favorecedora da exposição a menos eventos de risco para a criança (Crestani, Mattana, Moraes, \& Souza, 2013). Por outro lado, verifica-se na literatura achados diversos, desde a não influência direta do estado civil no comportamento das crianças (Leme \& Marturano, 2014), até a influência do divórcio no ajustamento da criança (Lucas, Nicholson, \& Erbas, 2013).

Com relação às condições pessoais dos pais abordar-se-á especificamente a depressão materna, dado seu reconhecido impacto negativo para os desfechos desenvolvimentais das crianças em todas as idades (Hayden \& Mash, 2014), sendo identificada como variável que interfere nas práticas educativas, sobretudo nas negativas (Trepat et al., 2014). No período pré-escolar e escolar, a depressão materna associada a outros eventos estressores relativos às práticas parentais, aos conflitos familiares e a condição socioeconômica das famílias têm sido consideradas relevantes para a predição de problemas de comportamento das crianças (Pizeta, Silva, Cartafina, \& Loureiro, 2013). Com base em uma robusta metanálise, Goodman et al. (2011) apontaram que a depressão materna tem sido relacionada à presença de problemas de internalização, externalização, psicopatologia geral e afeto negativo, além de menores níveis de afetos positivos.

O estudo sobre os problemas de comportamento na interface com práticas educativas é bastante investigado na literatura, quase sempre tendo como foco as variáveis preditoras dos problemas de comportamento, abordando pouco as variáveis contextuais e pessoais específicas que podem interferir no uso por parte das 
mães de práticas positivas e negativas. No presente estudo, abordar-se-á tal lacuna, ao se considerar como foco as práticas educativas maternas (práticas positivas e negativas), buscando-se identificar a influência para o uso dessas práticas das variáveis sociodemográficas, contextuais do ambiente familiar, da saúde mental materna e das variáveis comportamentais e demográficas das crianças. Objetiva-se comparar as práticas parentais relatadas pelas mães de crianças pré-escolares e escolares considerando as variáveis: demográficas das famílias, a diversidade de interações mãe-criança, características do relacionamento conjugal, indicadores de depressão materna, problemas de comportamento e habilidades sociais das crianças.

\section{Método}

\section{Participantes}

O estudo foi desenvolvido com uma amostra de conveniência de 151 mães biológicas e suas crianças, sendo 77 pré-escolares e 74 escolares, identificadas em 12 escolas municipais de educação infantil e 17 escolas de ensino fundamental, de uma cidade de médio porte do estado de São Paulo. Dessas 151 crianças, $47(31,1 \%)$ eram filhos únicos, 62 tinham um irmão (41,1\%), 31 tinham dois irmãos $(20,5 \%)$ e 11 tinham três ou mais irmãos (7,3\%). Foi incluída no estudo apenas uma criança por família. Tais crianças foram indicadas por seus respectivos professores, tendo por base a solicitação direta de identificação de crianças com a presença e a ausência de indicadores de problemas de comportamento. Foram convidadas 192 famílias de pré-escolares e 234 de escolares, totalizando 426 convites. Não aceitaram participar familiares de 96 de pré-escolares e de 135 de escolares, alegando predominantemente falta de interesse e/ou de tempo. No total, 96 familiares de pré-escolares e 99 de escolares responderam aos instrumentos, perfazendo um total de 195 participantes; destes participantes 44 foram excluídos do estudo por serem pais (21), avós (13) e tias (10), em função dos objetivos que envolvia avaliação de mães. Assim, foram incluídas 151 mães biológicas e a amostra ficou composta por 77 mães de pré-escolares (38 com indicativos de problemas e 39 sem indicativos) e por 74 de escolares (41 com indicativos e $33 \mathrm{sem}$ indicativos). Ainda que os professores tenham indicado as crianças, tal critério foi utilizado apenas para o acesso às famílias, já que a inclusão das crianças no estudo teve por suporte a avaliação materna por meio de instrumento específico e, com base no escore obtido, as crianças foram categorizadas como tendo ou não indicativos de problemas.

Com relação às variáveis demográficas, as crianças pré-escolares tinham idade média de 3,79 anos $(D P=$ $1,13)$ e as escolares de 8,18 anos $(D P=1,56)$. A amostra de pré-escolares foi composta por 42 meninos e 35 meninas, e a de escolares foi composta por 48 meninos e 26 meninas, sendo que o teste Qui-Quadrado não identificou diferenças quanto à distribuição por sexo em ambas as amostras $\left(X^{2}=1,669 ; p=0,196\right)$. A distribuição de crianças pré-escolares com e sem problemas de comportamento foi de 38 com indicativos de problemas e 39 sem indicativos e, quanto aos escolares, foram $41 \mathrm{com}$ indicativos e de $33 \mathrm{sem}$ indicativos de problemas de comportamento. De modo a avaliar possível viés quanto à composição dos grupos, procedeu-se a comparação deles com base no teste Qui-Quadrado e não se identificou diferenças significativas nessa distribuição amostral $\left(X^{2}=0,555 ; p=0,456\right)$.

Quanto às características demográficas das mães, tem-se: (a) a média de idade foi de 32,47 (DP = 6,33); (b) quanto ao estado civil, $79 \%$ delas eram casadas ou mantinham união estável, 13,2\% solteiras, $7 \%$ viúvas e $7,3 \%$ divorciadas; (c) no que se refere à escolaridade, $35,6 \%$ tinham primeiro grau (completo ou incompleto), $45 \%$ referiram ter o segundo grau (completo ou incompleto) e 18,5\%, o terceiro grau (completo ou incompleto); e (e) quanto ao trabalho remunerado, $49 \%$ relataram trabalhar fora de casa. A renda familiar, em salários mínimos teve a seguinte distribuição: 17,2\% até um salário; 29,1\% - dois salários mínimos; 27,9\% - três salários mínimos; $10,6 \%$ - quatro salários; $6,6 \%$ - cinco salários e 6,6\% acima de seis salários.

\section{Instrumentos}

a) Roteiro de Entrevista de Habilidades Sociais Educativas Parentais (RE-HSE-P, Bolsoni-Silva et al., 2016; Bolsoni-Silva \& Loureiro, 2010), é uma entrevista semi-estruturada que descreve a interação estabelecida entre pais e crianças, para tanto há medidas dos comportamentos parentais (práticas positivas e negativas), das crianças (queixas de problemas e habilidades sociais) e variáveis contextuais (por exemplo, se a conversação da mãe com a criança ocorre em diversos momentos do dia). As práticas educativas são relacionadas à comunicação, ao estabelecimento de limites e à expressão de sentimentos, classificadas em positivas (por exemplo, conversar sobre assuntos de interesse da criança, expressar afeto, solicitar mudança de comportamento) ou negativas (por exemplo, bater, 
xingar, gritar para estabelecer limites). O instrumento também prevê a avaliação de aspectos do relacionamento conjugal voltados para a educação dos filhos, incluindo consistência parental e agressividade conjugal. Precedendo as questões específicas, foram coletadas informações sobre: escolaridade, estado civil, nível socioeconômico e trabalho externo. $\mathrm{O}$ alfa para a amostra deste estudo foi de 0,846 e possui dois fatores, um que agrega aspectos positivos da interação (habilidades sociais educativas, habilidades sociais infantis e variáveis contextuais) e negativos (práticas negativas e problemas de comportamento), o primeiro fator teve o alfa de 0,827 e o segundo, de 0,646 . O instrumento diferencia crianças com e sem problemas de comportamento, tendo o CBCL Child Behavior Checklist (Inventário de Comportamentos da Infância e Adolescência, Achenbach \& Rescorla, 2001) como referência em análises de curva ROC e prevê indicações clínicas e não clínicas tanto para a diversidade como para a frequência comportamental de práticas educativas e comportamentos infantis. Para a avaliação de práticas parentais positivas a área foi de $0,769(p=0,001)$, com erro padrão de 0,065 e intervalo de confiança entre 0,641 e 0,896; para as habilidades sociais infantis a área encontrada foi de $0,734(p=0,003)$, com erro padrão de 0,065 e intervalo de confiança entre 0,599 e 0,869 (Bolsoni-Silva et al., 2016).

CBCL Child Behavior Checklist (Inventário de Comportamentos da Infância e Adolescência, Achenbach \& Rescorla, 2001) para pré-escolares e escolares (4 a 18 anos) que investiga, a partir do relato de familiares, a frequência de 113 respostas indicativas de problemas de comportamento. Os resultados são organizados em problemas internalizantes, externalizantes e totais, além de subscalas de problemas/ transtornos. Estudos psicométricos identificaram critérios satisfatórios de teste-positividade e de morbidade para os perfis clínico e não clínico (Bardin, Mari, \& Caeiro, 2003). Esse instrumento foi utilizado como medida de problema de comportamento, tomado por referência para diferenciar crianças com e sem problemas, adotando-se por critério para a presença de problemas de comportamento apresentar classificação clínica e/ou limítrofe em pelo menos uma das escalas (internalizante, externalizante, total).

Questionário de Respostas Socialmente Habilidosas para Pais (QRSH-Pais), avalia a frequência de respostas socialmente habilidosas, segundo relatos de familiares (Bolsoni-Silva, Marturano, \& Loureiro, 2011). O instrumento, com alfa de 0,82 , discriminou crianças com e sem problemas de comportamento. Neste estudo, esse instrumento foi utilizado como medida das habilidades sociais das crianças, tendo-se adotado como critério para recursos de habilidades ter pelo menos 25 pontos.

PHQ-9 - Questionário Sobre a Saúde do Paciente-9 (Patient Health Questionnaire). O PHQ-9 é um módulo baseado nos critérios para Desordem de Depressão Maior do DSM-IV. Ele foi proposto e validado por Kroenke, Spitzer e Williams (2001) que possibilita tanto o rastreamento de sinais e sintomas de depressão maior atual. No Brasil, foi conduzido um estudo psicométrico com o PHQ-9 (Osório, Mendes, Crippa, \& Loureiro, 2009), sendo a Entrevista Clínica Estruturada para o DSM - IV utilizada como instrumento padrão ouro, a qual apresenta uma excelente validade. Os resultados identificados foram de uma área sob a curva ROC (AUC) de 0,998 ( $p<0,001)$ e a nota de corte maior ou igual a 10 mostrou-se a mais adequada para rastreamento da depressão, com sensibilidade (S) de 1,00, especificidade (E) de 0,98, valor preditivo positivo de 0,97 , valor preditivo negativo de 1,00 e eficácia diagnóstica de 0,999 (Osório et al., 2009). Esse instrumento foi utilizado como medida de depressão, adotando-se como nota de corte o escore maior ou igual a10 como indicador de depressão.

\section{Procedimentos de Coleta de Dados}

\section{Aspectos Éticos}

Este projeto foi aprovado pelo Comitê de Ética em Pesquisa da universidade em que foi desenvolvido; sendo parte de um projeto maior com o título Saúde, Habilidades Sociais Conjugais e Educativas Parentais: Comparações quanto à escolaridade, ao gênero e a problemas de comportamento.

Após a aprovação pela Secretaria de Educação Infantil foram contatadas 17 escolas de Educação Infantil e 12 de ensino fundamental. Nesses contatos, foram apresentados os objetivos do estudo para a diretora ou para coordenadora pedagógica e com a concordância destas, em seguida, contataram-se as professoras, que, ao aceitarem participar, assinaram um Termo de Consentimento Livre e Esclarecido. Contou-se com a anuência de 38 professores de pré-escolares e de 37 de escolares, sendo solicitado a estes que indicassem duas crianças, da sala sob sua responsabilidade, uma que consideravam ter problemas de comportamento e outra sem problemas de comportamento. Na pré-escola, uma professora avaliou apenas uma criança. 
Com base nessas informações, 195 famílias de crianças indicadas pelos professores foram convidadas e aceitaram participar da pesquisa. Os familiares que aceitaram participar assinaram um Termo de Consentimento Livre e Esclarecido. As entrevistas foram conduzidas face a face, por entrevistador experiente e treinado para esta pesquisa, em locais de escolha das familias por lhes dar facilidade de acesso e privacidade (na própria casa, na escola ou universidade), momento em que responderam aos instrumentos em duas sessões de aplicação de aproximadamente uma hora cada (os instrumentos foram aplicados na ordem que foi descrito no método).

\section{Procedimento de Tratamento e Análise de Dados}

O tratamento dos dados foi conduzido de forma a identificar variáveis que pudessem estar correlacionadas na forma como as mães interagem com suas crianças, seja pelas práticas positivas (HSE-P), seja pelas negativas, as quais, de acordo com RE-HSE-P, foram identificadas como tendo indicadores de déficits em habilidades sociais educativas ou tendo indicadores de excessos de práticas negativas de educação. O RE-HSE-P permite identificar indicadores para déficits de práticas positivas e excessos de práticas negativas, os quais foram utilizados como critérios para compor os grupos. De acordo com os estudos psicométricos, o escore abaixo de sete para práticas positivas é tido como déficit. Quanto às práticas negativas, escores acima de sete são considerados como excessos. Nas comparações, foram consideradas para as análises as seguintes medidas: (a) dos comportamentos infantis a partir de dois instrumentos, seja para problemas de comportamento (CBCL, Queixas do RE-HSE-P), seja para habilidades sociais (QRSH, RE-HSE-P); (b) das práticas parentais, positivas e negativas (RE-HSE-P); (c) Diversidade de Contextos do RE-HSE-P); (d) da depressão materna (PHQ-9); (e) do relacionamento conjugal, que inclui consistência e agressividade (RE-HSE-P), cujas análises foram conduzidas com 119 participantes que eram casadas e/ou tinham união estável; e (f) variáveis demográficas das mães, a saber, escolaridade, renda e número de crianças que convivem na família. A escolaridade da criança foi controlada, uma vez que as análises foram conduzidas separadamente para pré-escolares e escolares. Em todos os testes foi considerada a significância de 5\%. Para verificar o tamanho do efeito quanto às significâncias nas comparações de grupo (teste $t$ amostras independentes), foram calculados os valores do d de Cohen (Cohen, 1988).

\section{Resultados}

Esta seção apresenta os dados das comparações quanto a variáveis que diferenciam grupos de mães que referiram usar práticas educativas positivas e negativas, tanto para pré-escolares, como para escolares. Em um primeiro momento, apresentam-se os achados para as práticas positivas de pré-escolares (Tabela 1) seguida das práticas positivas dos escolares (Tabela 2). Na sequência, encontram-se os correspondentes às práticas negativas de pré-escolares (Tabela 3), e dos escolares (Tabela 4).

De acordo com a Tabela 1, nota-se que as mães de pré-escolares que apresentaram indicadores para problemas quanto às práticas parentais positivas (habilidades sociais educativas - HSE) usavam menos diversidade e frequência de habilidades sociais educativas e referiram menor diversidade de contextos de interação social com as crianças. Os filhos, por sua vez, tinham menos habilidades sociais e mais queixas de problemas de comportamento. Por outro lado, as mães que referiram fazer uso de práticas positivas, com diversidade e frequência compatíveis a não ter problemas, conversavam, faziam perguntas e estabeleciam regras com mais frequência, bem como ouviam o cônjuge, mudavam de comportamento e se desculpavam com o cônjuge. Essas mães sem dificuldades quanto às práticas positivas também relataram, mais frequentemente, sentirem-se bem. Quanto às medidas de efeito, verificou-se valores acima de 0,8 no $d$ de Cohen, para as diversidades e frequências de hse, a diversidade de interações sociais estabelecidas com a criança, as habilidades sociais infantis e a prática positiva de estabelecer regras, caracterizando um efeito grande. Como efeito médio, destacaram-se as práticas positivas de dar explicações, perguntar/conversar, mudar de comportamento/desculpar-se com o cônjuge e sentir-se bem. Queixas de problemas de comportamento e ouvir opinião do cônjuge tiveram medida de efeito próximo ao valor médio (maior ou igual a 0,5 ).

Quanto às variáveis sociodemográficas, apenas a escolaridade materna diferenciou os grupos, indicando que as mais habilidosas apresentavam mais instrução formal. Destaca-se que as práticas negativas não diferenciaram os grupos, ou seja, por esses achados não se pode afirmar que as mães que têm mais dificuldades em usar práticas positivas, usavam com maior frequência as práticas negativas. $\mathrm{O}$ mesmo ocorreu quanto aos indicadores de depressão materna.

Considerando a Tabela 2, nota-se que, para as mães de crianças escolares quanto à avaliação das 
Tabela 1.

Comparaçoes, com Significância Estatística, Relativas às Práticas Positivas (HSE) de Mães de Pré-Escolares

\begin{tabular}{|c|c|c|c|c|c|}
\hline \multirow[t]{3}{*}{ Categorias } & $\begin{array}{l}\text { Com indicador } \\
\quad(n=43)\end{array}$ & $\begin{array}{c}\text { Sem } \\
\text { indicador } \\
(n=34)\end{array}$ & & & \\
\hline & Média (Desvi & padrão) & $t$ & $p$ & $d$ Cohen \\
\hline & \multicolumn{5}{|c|}{ Variáveis numéricas } \\
\hline Escore Práticas Positivas - HSE- diversidade & $7,35(1,69)$ & $\begin{array}{l}12,47 \\
(2,50)\end{array}$ & 10,701 & 0,000 & 2,49 \\
\hline Escore Práticas Positivas - HSE- freq. & $8,47(2,64)$ & $\begin{array}{l}10,82 \\
(2,15)\end{array}$ & 4,216 & 0,000 & 0,98 \\
\hline Diversidade de interações & $9,30(4,15)$ & $\begin{array}{l}12,85 \\
(4,09)\end{array}$ & 3,752 & 0,000 & 0,87 \\
\hline Habilidades sociais infantis & $10,42(3,03)$ & $\begin{array}{l}14,26 \\
(4,98)\end{array}$ & 4,184 & 0,000 & 0,97 \\
\hline Queixas de problemas de compto & $0,44(0,85)$ & $0,12(0,41)$ & $-2,035$ & 0,045 & 0,47 \\
\hline Práticas Positivas: Dá explicações & $0,35(0,57)$ & $0,82(0,87)$ & 2,879 & 0,005 & 0,66 \\
\hline Práticas Positivas: Pergunta/conversa & $1,47(1,28)$ & $2,32(1,01)$ & 3,205 & 0,002 & 0,73 \\
\hline Práticas Positivas: Estabelece regras & $0,19(0,39)$ & $0,74(0,90)$ & 3,601 & 0,001 & 0,84 \\
\hline Práticas Positivas: Sente-se bem & $0,49(0,70)$ & $0,94(0,65)$ & 2,904 & 0,005 & 0,67 \\
\hline Relacionamento conjugal: Ouve opinião conj. & $0,67(0,71)$ & $1,06(0,98)$ & 1,986 & 0,051 & 0,48 \\
\hline \multirow[t]{2}{*}{$\begin{array}{l}\text { Relacionamento conjugal: Muda compto/ } \\
\text { desculpa-se cônjuge }\end{array}$} & $0,23(0,43)$ & $0,50(0,66)$ & 2,143 & 0,035 & 0,51 \\
\hline & \multicolumn{5}{|c|}{ Variáveis categóricas } \\
\hline Categorias & Subcategorias & $\begin{array}{c}\text { Com } \\
\text { indicador }\end{array}$ & $\begin{array}{c}\text { Sem } \\
\text { indicador }\end{array}$ & $\mathrm{X}^{2}$ & $p$ \\
\hline \multirow[t]{2}{*}{ Escolaridade } & Até 8 anos & 39 & 24 & 5,161 & 0,023 \\
\hline & Mais de 8 anos & 4 & 10 & & \\
\hline \multirow[t]{2}{*}{ Habilidades sociais- qrsh } & clínico & 13 & 4 & 3,764 & 0,052 \\
\hline & não clínico & 30 & 30 & & \\
\hline \multirow[t]{2}{*}{ Práticas positivas: HSE- freq. } & clínico & 40 & 26 & 4,249 & 0,039 \\
\hline & não clínico & 3 & 8 & & \\
\hline
\end{tabular}

práticas parentais positivas, um número maior de itens diferenciou os grupos. No grupo com dificuldades em utilizar práticas positivas, foram menos frequentes a diversidade e frequência de habilidades sociais educativas, habilidades sociais infantis e variáveis de contexto, tal como na amostra de pré-escolares. Adicionalmente, para essas mães, verificou-se maior escore e classificação de depressão e também relataram não concordarem com a opinião do cônjuge. Quanto a práticas específicas, as mães sem dificuldades referiram oferecer aos filhos mais explicações e carinho, relatando sentirem-se bem. Também relataram mudar de comportamento e se desculparem com o cônjuge, quando necessário e, sobretudo, que pensavam de maneira concordante. Por meio das medidas de efeito, verificou-se efeito grande para as HSE (diversidade e frequência), diversidade de interações sociais com a criança, relato espontâneo sobre as habilidades sociais das crianças (RE-HSE-P), prática positiva de fazer carinho e sentir-se bem; e medidas de efeito médio para escores de depressão, para as habilidades sociais infantis com o instrumento dirigido (QRSH-Pais), as práticas positivas de conversar 
Tabela 2

Comparacões, com Significância Estatística, Relativas às Práticas Positivas (HSE) de Mães de Escolares

\begin{tabular}{|c|c|c|c|c|c|}
\hline \multirow{3}{*}{ Categorias } & $\begin{array}{l}\text { Com indicador } \\
\quad(n=39) \\
\end{array}$ & $\begin{array}{c}\text { Sem indicador } \\
\quad(n=35)\end{array}$ & & & \\
\hline & \multicolumn{2}{|c|}{ Média (Desvio padrão) } & $t$ & $p$ & $d$ de Cohen \\
\hline & \multicolumn{5}{|c|}{ Variáveis numéricas } \\
\hline $\begin{array}{l}\text { Escore Práticas Positivas - HSE - } \\
\text { diversidade }\end{array}$ & $6,67(2,07)$ & $12,51(2,93)$ & 9,804 & 0,000 & 2,35 \\
\hline $\begin{array}{l}\text { Escore Práticas Positivas - } \\
\text { HSE- freq. }\end{array}$ & $7,62(2,66)$ & $10,43(2,33)$ & 4,848 & 0,000 & 1,13 \\
\hline Diversidade de interações & $8,62(4,00)$ & $14,49(6,09)$ & 4,950 & 0,000 & 1,17 \\
\hline Habilidades sociais infantis & $8,15(3,77)$ & $12,77(4,09)$ & 5,035 & 0,000 & 1,19 \\
\hline Habilidades sociais infantis - QRSH & $28,62(6,08)$ & $32,14(3,60)$ & 2,992 & 0,004 & 0,73 \\
\hline Escore depressão & $7,67(7,54)$ & $3,43(4,01)$ & $-2,967$ & 0,004 & 0,70 \\
\hline Práticas Positivas: Conversa & $1,82(0,38)$ & $2,00(0,00)$ & 2,729 & 0,008 & 0,66 \\
\hline Práticas Positivas: Dá explicações & $0,23(0,43)$ & $0,54(0,66)$ & 2,447 & 0,017 & 0,57 \\
\hline Práticas Positivas: Faz carinhos & $3,36(1,99)$ & $5,57(2,58)$ & 4,092 & 0,000 & 0,98 \\
\hline Práticas Positivas: Sente-se bem & $0,33(0,53)$ & $0,94(0,97)$ & 3,405 & 0,001 & 0,80 \\
\hline $\begin{array}{l}\text { Relacionamento conjugal: Muda/ } \\
\text { desculpa-se conj. }\end{array}$ & $0,18(0,39)$ & $0,66(0,94)$ & 2,916 & 0,005 & 0,69 \\
\hline $\begin{array}{l}\text { Relacionamento conjugal: Casal tem } \\
\text { pensamento concordante }\end{array}$ & $0,10(0,31)$ & $0,29(0,46)$ & 2,037 & 0,045 & 0,49 \\
\hline \multirow[t]{2}{*}{$\begin{array}{l}\text { Relacionamento conjugal: Casal tem } \\
\text { pensamento discordante }\end{array}$} & $0,62(0,94)$ & $0,26(0,56)$ & $-1,970$ & 0,053 & 0,46 \\
\hline & \multicolumn{3}{|c|}{ Variáveis categóricas } & & \\
\hline Categorias & Subcategorias & Com indic. & Sem indic. & $\mathrm{X}^{2}$ & $p$ \\
\hline \multirow[t]{2}{*}{ Escolaridade } & Até 8 anos & 36 & 22 & 10,332 & 0,001 \\
\hline & Mais de 8 anos & 2 & 13 & & \\
\hline \multirow[t]{2}{*}{ Renda } & até 3 salários & 34 & 19 & 16,319 & 0,012 \\
\hline & de 4 a 7 salários & 3 & 15 & & \\
\hline \multirow[t]{2}{*}{ Habilidades sociais - QRSH } & clínico & 12 & 1 & 9,924 & 0,002 \\
\hline & não clínico & 27 & 34 & & \\
\hline \multirow[t]{2}{*}{ Clas. Práticas Positivas - HSE- freq. } & clínico & 38 & 29 & 4,578 & 0,032 \\
\hline & não clínico & 1 & 6 & & \\
\hline \multirow[t]{2}{*}{ Clas. Depressão } & clínico & 14 & 3 & 7,784 & 0,005 \\
\hline & não clínico & 32 & 25 & & \\
\hline
\end{tabular}

e dar explicações e os itens de relacionamento conjugal (muda de opinião, desculpa-se, concorda e discorda). Parte das comparações apresentou medida de efeito grande (diversidade e frequência no uso de prática negativa, bater e sentir-se mal após estabelecer limites) e parte, efeito médio (queixas de problemas de comportamento, diversidade das interações, conversar, expressar sentimento negativo, colocar de castigo, gritar/ficar 
bravo/xingar, considerar o cônjuge agressivo e discordar do cônjuge).

No que se refere às variáveis sociodemográficas, tanto escolaridade materna quanto a renda familiar diferenciaram os grupos, tendo o grupo sem problemas para práticas positivas mais instrução e renda. Quanto às práticas negativas, os achados convergem com os da idade pré-escolar, não diferenciando os grupos com ou sem dificuldades em utilizar práticas positivas.

De acordo com a Tabela 3, verifica-se que as mães de pré-escolares que relataram utilizar excessivamente práticas negativas, indicaram mais frequência e diversidade dessas práticas, sobretudo, castigar, bater, gritar/ ficar brava/xingar. Nota-se também que relataram conversar menos e expressarem mais sentimentos negativos, que estão relacionados, sobretudo, às práticas negativas de bater e/ou gritar, além de avaliarem o cônjuge como agressivo e pensarem de forma diferente deste, ainda, essas mães referiram sentir-se mal com mais frequência.

Quanto às variáveis demográficas, apenas o número de irmãos diferenciou os grupos, sendo que a presença de um irmão ocorreu com mais frequência no grupo que relatou utilizar excessivamente práticas negativas para educar os filhos. Destaca-se que, enquanto categoria geral as práticas positivas não diferenciaram os grupos, mas em comportamentos específicos foram identificadas diferenças, sugerindo que as mães com

Tabela 3.

Comparaçôes, com Significância Estatística, Relativas às Práticas Negativas de Mães de Pré-Escolares

\begin{tabular}{|c|c|c|c|c|c|}
\hline \multirow{3}{*}{ Categorias } & $\begin{array}{l}\text { Com indicador } \\
\quad(n=44)\end{array}$ & $\begin{array}{l}\text { Sem indicador } \\
\quad(n=33)\end{array}$ & & & \\
\hline & \multicolumn{2}{|c|}{ Média (Desvio padrão) } & $t$ & $p$ & $d$ de Cohen \\
\hline & \multicolumn{5}{|c|}{ Variáveis numéricas } \\
\hline Escore Prática negativa diversidade & $8,52(2,33)$ & $3,03(1,49)$ & $-11,848$ & 0,000 & 2,76 \\
\hline Escore Prática negativa frequência & $7,45(3,16)$ & $4,09(2,61)$ & $-4,971$ & 0,000 & 1,16 \\
\hline Problemas de comportamento & $8,41(4,06)$ & $5,45(3,64)$ & $-3,301$ & 0,001 & 0,78 \\
\hline Diversidade de interações & $0,68(0,86)$ & $0,21(0,48)$ & $-2,826$ & 0,006 & 0,66 \\
\hline Práticas Positivas: Conversa & $1,89(0,32)$ & $2,00(0,00)$ & 2,030 & 0,046 & 0,46 \\
\hline $\begin{array}{l}\text { Práticas Negativas: Sentimento } \\
\text { negativo }\end{array}$ & $1,23(0,74)$ & $0,55(0,62)$ & $-4,279$ & 0,000 & 0,99 \\
\hline Práticas Negativas: Coloca de castigo & $0,73(0,87)$ & $0,21(0,48)$ & $-3,054$ & 0,003 & 0,72 \\
\hline Práticas Negativas: Bate & $0,70(0,88)$ & $0,09(0,29)$ & $-3,852$ & 0,000 & 0,89 \\
\hline $\begin{array}{l}\text { Práticas Negativas: Grita/fica brava/ } \\
\text { xinga }\end{array}$ & $0,66(0,83)$ & $0,21(0,48)$ & $-2,749$ & 0,007 & 0,65 \\
\hline Sente-se mal após estabelecer limites & $20,64(10,46)$ & $10,76(10,23)$ & $-2,791$ & 0,007 & 0,97 \\
\hline $\begin{array}{l}\text { Relacionamento conjugal: Considera } \\
\text { cônjuge agressivo }\end{array}$ & $0,59(10,13)$ & $0,03(0,17)$ & $-2,827$ & 0,006 & 0,07 \\
\hline \multirow[t]{2}{*}{$\begin{array}{l}\text { Relacionamento conjugal: Casal tem } \\
\text { pensamento discordante }\end{array}$} & $0,61(0,65)$ & $0,30(0,47)$ & $-2,318$ & 0,023 & 0,54 \\
\hline & \multicolumn{2}{|c|}{ Variáveis categóricas } & & & \\
\hline Categorias & Subcategorias & Com indicador & $\begin{array}{l}\text { Sem } \\
\text { indicador }\end{array}$ & $X^{2}$ & $p$ \\
\hline \multirow[t]{3}{*}{ Número de crianças } & 1 criança & 12 & 16 & & \\
\hline & 2 crianças & 24 & 8 & 7,206 & 0,027 \\
\hline & 3 ou mais & 8 & 9 & & \\
\hline \multirow[t]{2}{*}{ Clas. pr. neg.- freq. } & clínico & 6 & 0 & 4,880 & 0,027 \\
\hline & não clínico & 38 & 33 & & \\
\hline
\end{tabular}


excessos de práticas negativas apresentavam mais déficits em práticas positivas (HSE-P). Adicionalmente, verificou-se que a utilização de práticas negativas com mais frequência não diferenciou as habilidades sociais infantis, e também que a depressão materna não diferenciou os grupos.

Para as mães de escolares nas comparações relativas às práticas negativas, os achados são semelhantes aos de pré-escolares. Elas relataram mais frequência e diversidade de práticas negativas, sobretudo, ameaçar, castigar, bater, gritar/ficar brava/xingar. Notou-se também que referiram conversar menos, expressarem mais sentimentos negativos e relataram ter mais dificuldade de cumprir promessas. Nesse grupo, as mães também avaliaram os cônjuges como mais agressivos e referiram pensar de forma discordante deste, além de, com mais frequência, sentirem-se mal. As medidas de efeito foram grandes para a maioria dos itens avaliados (frequência e diversidade de práticas negativas, queixas de problemas de comportamento, dificuldade em cumprir promessas, colocar de castigo, gritar/ficar bravo/xingar, sentir-se mal, considerar cônjuge agressivo e discordar dele. Com medidas de efeito médio, identificou-se as comparações relativas as habilidades sociais infantis, conversar, identificar comportamento que desaprova e bater.

Tabela 4.

Comparações, com Significância Estatística, Relativas às Práticas Negativas de Mães de Escolares

\begin{tabular}{|c|c|c|c|c|c|}
\hline \multirow{3}{*}{ Categorias } & $\begin{array}{c}\text { Com indicador } \\
\quad(n=35)\end{array}$ & $\begin{array}{c}\text { Sem indicador } \\
\quad(n=39)\end{array}$ & & & \\
\hline & \multicolumn{2}{|c|}{ Média (Desvio padrão) } & $t$ & $p$ & $d$ de Cohen \\
\hline & \multicolumn{5}{|c|}{ Variáveis numéricas } \\
\hline Escore Prática negativa diversidade & $9,51(2,50)$ & $3,28(1,41)$ & $-13,368$ & 0,000 & 3,16 \\
\hline Escore Prática negativa frequência & $7,94(3,32)$ & $3,87(2,31)$ & $-6,170$ & 0,000 & 1,46 \\
\hline Diversidade de interações & $8,74(2,85)$ & $0,36(0,67)$ & $-2,876$ & 0,006 & 4,20 \\
\hline Habilidades sociais infantis & $7,20(2,82)$ & $5,87(2,66)$ & $-2,080$ & 0,041 & 0,49 \\
\hline Problemas de comportamento & $8,17(4,09)$ & $4,64(3,14)$ & $-4,129$ & 0,000 & 0,99 \\
\hline Práticas Positivas: Conversa & $1,83(0,38)$ & $1,97(0,16)$ & 2,179 & 0,033 & 0,49 \\
\hline Tem dificuldade em cumprir promessas & $0,80(0,72)$ & $0,33(0,58)$ & $-3,055$ & 0,003 & 0,73 \\
\hline $\begin{array}{l}\text { Práticas Negativas: Identifica comptos } \\
\text { que não gosta }\end{array}$ & $1,57(0,61)$ & $1,08(0,84)$ & $-2,873$ & 0,005 & 0,67 \\
\hline Práticas Negativas: Ameaça & $0,37(0,65)$ & $0,03(0,16)$ & $-3,238$ & 0,002 & 0,74 \\
\hline Práticas Negativas: Coloca de castigo & $0,40(0,55)$ & $0,13(0,34)$ & $-2,578$ & 0,012 & 0,60 \\
\hline Práticas Negativas: Bate & $0,46(0,66)$ & $0,08(0,27)$ & $-3,317$ & 0,001 & 0,78 \\
\hline $\begin{array}{l}\text { Práticas Negativas: Grita/fica brava/ } \\
\text { xinga }\end{array}$ & $1,17(1,22)$ & $0,33(0,62)$ & $-3,770$ & 0,000 & 0,89 \\
\hline Sente-se mal após estabelecer limites & $2,34(1,21)$ & $1,26(1,23)$ & $-3,825$ & 0,000 & 0,89 \\
\hline $\begin{array}{l}\text { Relacionamento conjugal: Considera } \\
\text { cônjuge agressivo }\end{array}$ & $0,63(1,21)$ & $0,13(0,41)$ & $-2,425$ & 0,018 & 1,04 \\
\hline Casal tem pensamento discordante & $0,83(0,98)$ & $0,10(0,31)$ & $-4,376$ & 0,000 & 0,89 \\
\hline \multicolumn{6}{|c|}{ Variáveis categóricas } \\
\hline Categorias & Subcategorias & Com indic. & Sem indic. & $\mathrm{X}^{2}$ & $p$ \\
\hline \multirow[t]{2}{*}{ Probl. compto. cbcl. } & clínico & 24 & 17 & 4,659 & 0,031 \\
\hline & não clínico & 11 & 22 & & \\
\hline \multirow[t]{2}{*}{ Clas. prat neg. - freq. } & clínico & 10 & 0 & 12,884 & 0,000 \\
\hline & não clínico & 25 & 39 & & \\
\hline
\end{tabular}


O grupo que referiu fazer mais uso de práticas negativas identificou, por relato espontâneo (RE-HSE-P), mais comportamentos que as desagradavam e, suas crianças, apresentaram mais problemas de comportamento pela avaliação do CBCL. Diferentemente da idade pré-escolar, as crianças do período escolar que conviviam com mais práticas negativas, apresentaram menores escores de habilidades sociais e as variáveis sociodemográficas e a depressão materna não diferenciaram os grupos.

\section{Discussão}

A interação estabelecida entre mães e filhos é resultado de múltiplos fatores que se influenciam mutuamente. A presença de problemas de comportamento (Lins, Alvarenga, Santos, Almeida, \& Santos, 2012), depressão materna (Trepat et al., 2014; Hayden \& Mash, 2014), conflitos conjugais (Lucas et al., 2013; Pizeta et al., 2013) e determinadas variáveis sociodemográficas, como sexo, escolaridade da criança, sexo do respondente e estado civil (Berry \& O'Connor, 2010; Lucas et al., 2013; Crestani et al.,2013) são consideradas condições que podem ter algum efeitos sobre as práticas parentais. No presente estudo, buscou-se avaliar simultaneamente tais variáveis, considerando as mães terem dificuldades no uso de práticas positivas e/ou relatarem usar excessivamente práticas negativas.

Sumariando, os achados da presente pesquisa permitem afirmar que mães de crianças pré-escolares e escolares sem dificuldades quanto ao uso de práticas positivas relataram que elas são mais habilidosas, além de terem menos queixas de problemas, o que é concordante com diversos estudos (Berry \& O'Connor, 2010; Borden et al., 2014; Kettler, Elliott, Davies, \& Griffi, 2011). Por outro lado, aquelas com déficits quanto ao uso de práticas positivas, tanto para crianças pré-escolares como para as escolares, relataram usar com menos frequência e diversidade comportamentos de interação social, o que é consonante com o relato de Bolsoni-Silva \& Loureiro, (2011). As crianças filhas de mães com déficits em práticas positivas eram as que tinham menos habilidades sociais e apresentavam mais queixas de problemas, como já relatado por estudos nacionais e internacionais (Leme \& Bolsoni-Silva, 2010; Kim, Doh, Hong, \& Choi, 2010; Marin et al., 2012). Tais achados têm respaldo na literatura, que aponta que o uso práticas positivas de educação se associa a melhores escores de habilidades sociais e a menos queixas de problemas de comportamento (Leme \& Bolsoni-Silva, 2010;
Marin et al., 2012), pois as habilidades podem se tornar funcionalmente equivalentes (Bolsoni-Silva \& Carrara, 2010). A diversidade de situações positivas de interação social, como brincadeiras, acompanhar a tarefa, conversar sobre diferentes temas e situações podem propiciar melhores interações sociais, dando à criança atenção e funcionando como um modelo de comportamentos socialmente habilidosos (Bolsoni-Silva \& Loureiro, 2011; Patterson, Reid, \& Dishion, 2002).

As variáveis que diferenciaram pré-escolares de escolares quanto às dificuldades no uso de práticas positivas foram a depressão materna e as características do relacionamento conjugal que dificultam manter consistência, tais como agressividade e opiniões divergentes (Leme \& Bolsoni-Silva, 2010). Esses achados indicam que a depressão materna e as dificuldades no relacionamento conjugal são condições que interferem nas práticas educativas positivas, sobretudo para os escolares no que tange à presença de depressão, ainda que outros estudos tenham apontado tal influência no uso de práticas negativas (Trepat et al., 2014), e não nas positivas. A depressão materna, pelas características clínicas do transtorno pode diminuir a responsividade à criança, favorecendo que atente menos para os comportamentos esperados e dê menos atenção para as interações diversas, em comparação a aquelas necessárias para estabelecer limites, o que de certo modo pode interferir na ocorrência de práticas positivas de educação.

Interessante destacar que a depressão diferenciou os grupos na idade escolar, mas não na pré-escolar, possivelmente, porque os escolares exigem mais dos pais, uma vez que as crianças apresentam maior variabilidade comportamental, o que pode favorecer mais questionamentos quanto à obediência; além disso, na idade escolar as exigências de desempenho acadêmico e social aumentam, contribuindo para uma maior ocorrência de comportamentos desafiadores. Tal interpretação tem respaldo nas afirmativas de Patterson et al. (2002) que referiram uma progressão de problemas de comportamento da pré-escola para a escola. De acordo com Hudson, Flannery-Schroeder e Kendall (2004) crianças que irritam mais os adultos estão em maior risco de serem alvo de práticas negativas. Os dados do presente estudo são concordantes com esses achados, pois as práticas negativas diferenciaram crianças/escolares com e sem problemas de comportamento.

Por outro lado, diversos estudos apontaram mais problemas de comportamento na pré-escola (BolsoniSilva et al., 2016) o que é discordante dos achados da 
presente pesquisa, que ocorreram com mais frequência na idade escolar associado ao uso de prática negativa. Uma hipótese adicional sobre tal questão é a de que as crianças/escolares precisam se adaptar as exigências do ensino formal, acadêmico, que também lhe exige mais, em comparação com o ensino infantil, que tem uma natureza mais lúdica (Buyse, Verschueren, Doumen, Damme, \& Maes, 2008). Segundo Patterson, et al. (2002) lidar com tarefas difíceis pode ser um motivo para a emissão de problemas de comportamento. Além disso, os pais podem ter mais paciência e autocontrole com as crianças menores, por considerarem que elas precisam de mais ajuda que as escolares.

Algumas práticas parentais positivas mais frequentes para quem não tinha dificuldade foram semelhantes para pré-escolares e escolares, tais como dar explicações, relatar sentir-se bem, mudar de comportamento e pedir desculpas. Entre os pré-escolares as mães conversavam, faziam perguntas e estabeleciam regras com mais frequência e entre os escolares as mães eram mais carinhosas. Tais comportamentos positivos já foram documentados na literatura (Gomide, 2006) como importantes para a promoção de habilidades sociais e prevenção de problemas de comportamento. Chama a atenção que os comportamentos que apareceram igualmente na pré-escola e escola parecem mais relacionados ao estabelecimento de limites, enquanto os de comunicação diferenciaram apenas na pré-escola e os de carinho/afeto, na idade escolar, indicando peculiaridades quanto à idade e as práticas. Tais achados sugerem que o uso de práticas positivas para estabelecer limites é sempre de suma importância, sem desconsiderar os comportamentos de comunicação e afeto que são mais ou menos importante de acordo com a idade das crianças (Bolsoni-Silva \& Loureiro, 2011). Diferenciando pré-escolares de escolares, as mães de escolares relataram ter mais dificuldade de cumprir promessas, além de conversarem menos com suas crianças, o que parece indicar que a interação com os filhos na idade escolar é mais difícil, exigindo mais das mães, o que tem respaldo a discussão prévia (Hudson et al., 2004; Patterson et al., 2002).

No que se refere às mães que usavam excessivamente práticas negativas, as de pré-escolares e de escolares relataram com mais frequência e diversidade que expressavam sentimentos negativos, além de castigar, bater, gritar, ficar brava e xingar, sendo que as mães de escolares, adicionalmente, relataram também ameaçar. Tais práticas são bem documentadas na literatura como preditoras de problemas de comportamento
(Price et al., 2013; Trepat et al., 2014; Xing \& Wang, 2013). Contudo, os dados mostraram poucas diferenças entre mães de pré-escolares e de escolares, o que é concordante com Bolsoni-Silva et al. (2016) que também não encontraram diferenças na pré-escola e ensino fundamental quanto aos problemas de comportamento. Patterson et al. (2002) também relataram a ocorrência de práticas negativas em famílias de crianças com e sem problemas de comportamento.

Em ambos os grupos identificou-se mães que relataram sentirem-se mal, além de terem relacionamento conjugal prejudicado, sendo o cônjuge descrito como agressivo e que pensava de maneira divergente delas, o que é concordante com o relatado por Bolsoni-Silva e Loureiro (2011). Tais dados sugerem a dificuldade na consistência quanto ao uso de práticas educativas parentais por mães e pais, o que pode deixar pouco claro para a criança quais as regras de convivência, bem como quais as consequências por cumprir ou não. Dessa forma as intervenções com famílias precisam ensinar, além de como estabelecer limites, ser afetuoso e comunicativo com as crianças, também a como interagir com o cônjuge, de forma a reduzir a agressividade e aumentar a consistência.

Para os pré-escolares, os escores de problemas de comportamento do RE-HSE-P e para os escolares os escores do CBCL diferenciaram os grupos, indicando que a presença de problemas de comportamento influenciou no uso de práticas negativas. Esse achado aponta para a bidirecionalidade das interações sociais, pois a literatura é clara ao afirmar que práticas negativas estimulam problemas de comportamento (Xing \& Wang, 2013), bem como as que crianças podem ser pouco sensíveis à punição (Hudson et al., 2004) e ao apresentarem comportamentos agressivos, opositivos (Patterson et al., 2002) acabam sendo punidas mais frequentemente por seus pais, que tentam por um lado com essas estratégias garantir a obediência e, por outro, também se sentem mais nervosos com dificuldade de se controlar e de usar preferencialmente, práticas positivas diante da desobediência recorrente (Patterson et al., 2002). O resultado de que as mães relataram se sentir mal quando usam práticas negativas sugere que realmente o fazem por não conseguirem serem positivas sempre que a criança desobedece e, ao contrário, quando usam práticas positivas sentem-se bem, confirmando achados de outras pesquisas (Bolsoni-Silva \& Loureiro, 2011).

Considerando nas comparações aquelas que apresentaram tamanho de efeitos grandes, notam-se 
peculiaridades quanto as idades e os tipos de práticas. Quanto à idade pré-escolar, destacam-se as habilidades sociais infantis e para práticas positivas, a diversidade e frequência no seu uso, de maneira geral e, em particular, o estabelecer regras; já quanto às práticas negativas, evidenciou-se a diversidade e frequência no seu uso e, em particular, o bater, a forma como expressa sentimento negativo (agressivo) e o sentir-se mal. Para os escolares, também destacaramse a frequência e diversidade de práticas positivas, a diversidade de contextos de interações, o fazer carinho e sentir-se bem, além das habilidades sociais infantis; quanto às práticas negativas, evidenciaram-se as queixas de problemas de comportamento, frequência e diversidade no uso dessas práticas, diversidade de interações, gritar/xingar, sentir-se mal e identificar cônjuge como agressivo e pensar diferente dele. Assim pode-se verificar que, para os pré-escolares, é mais crucial estabelecer regras e para os escolares, o fazer carinho, sendo mais prejudicial para os pré-escolares o bater, enquanto que, para os escolares, é o gritar/xingar e conviver com relacionamento conjugal conflitivo.

O ensino de habilidades sociais educativas (Bolsoni-Silva \& Marturano, 2010) em intervenções com pais pode ajudar a aprimorar o repertório para estabelecer limites de forma positiva, por um lado e, por outro, interagir com a criança em outros momentos que não apenas para ensinar certo e errado, garantindo assim afeto e comunicação, o que pode reduzir os problemas de comportamento. Vale destacar que com as crianças é necessário ensinar habilidades sociais para lidar com as demandas dos contextos familiar, escolar e com os pares, o que demanda aprender a obedecer e a seguir regras, tais pontos são relevantes para as intervenções (Falcão \& Bolsoni-Silva, 2015). Os dados na presente pesquisa demonstraram a necessidade de se avaliar simultaneamente comportamentos parentais e infantis, de forma a descrever as influências mútuas.

Quanto às variáveis sociodemográficas, a instrução formal diferenciou para ambas as escolaridades e a renda diferenciou para o ensino fundamental (Algarvio et al., 2013; Buehler \& Gerard, 2013). Considera-se que, quanto mais instrução formal a mãe tem, mais ela pode ter acesso a informações sobre a importância de interagir positivamente com a criança, criando condições de estimulação e de interação social positiva, assim como a renda familiar pode também propiciar o acesso a ambientes e materiais que estimulam o desenvolvimento positivo da criança. Apenas para os pré-escolares a presença de irmãos influenciou no uso de práticas negativas, o que parece concordar com Freitas e Piccinini (2010) que também encontrou o uso mais frequente de punição por mães com dois filhos ao serem comparadas com apenas um único filho. Crianças pré-escolares podem ser mais sensíveis à presença de um irmão no que tange a ter que dividir a atenção dos pais, podendo emitir mais comportamentos de oposição, desobediência na tentativa de obter atenção, aumentando a chance de uso de práticas negativas pelos pais.

\section{Considerações Finais}

Esta pesquisa comparou grupos de pré-escolares e de escolares variáveis comportamentais (depressão, relacionamento conjugal, comportamentos infantis) e demográficas (sexo, escolaridade, renda, número de filhos) que podem impactar no uso das práticas educativas positivas e negativas das mães. Identificou-se que mães de crianças com e sem problemas de comportamento utilizam tanto práticas positivas como negativas para educar seus filhos, sendo as práticas negativas mais frequentes para as crianças/escolares com problemas de comportamento. De maneira geral, as práticas positivas ocorreram com mais frequência quando as crianças se comportam de maneira habilidosa e, ao contrário, as negativas quando as crianças apresentaram problemas de comportamento, tanto para pré-escolares como para escolares. O relacionamento conjugal se mostrou variável importante em ambas às escolaridades e a depressão materna, apenas na idade escolar, influenciando nos déficits de habilidades sociais educativas. A escolaridade materna influenciou no uso de práticas para pré-escolares e escolares, enquanto que a renda diferenciou os déficits de práticas positivas apenas na idade escolar. O número de filhos diferenciou os grupos quanto ao uso de práticas negativas, apenas para as mães de pré-escolares.

Destacam-se como pontos fortes do estudo ter garantido distribuição equivalente de meninos e meninas pré-escolares e escolares, bem como na composição da amostra terem sido incluídas apenas mães biológicas e casadas ou em união consensual, além da utilização de instrumentos aferidos. Como limites desta pesquisa destacam-se o delineamento transversal, o uso exclusivo de medidas de relato que tiveram as mães como informantes e, com relação à depressão materna, a ausência de confirmação dos indicadores por diagnóstico clínico. 
Sugere-se que estudos futuros incluam, além do relato, medidas de observação, contem com outros informantes além das mães, como pais e crianças e ainda que avaliem amostras de crianças com características específicas quanto a déficits ou recursos desenvolvimentais. Os achados desta pesquisa podem contribuir para o planejamento de ações de prevenção e intervenção em programas de orientação de pais.

\section{Referências}

Achenbach, T. M., \& Rescorla, L. A. (2001). Manual for the ASEBA School-Age Forms \& Profiles. Burlington, VT: University of Vermont, Research Center for Children, Youth \& Families.

Algarvio, S., Isabel, L., \& Maroco, J. (2013). Parental concerns' prevalence and socio-demographic variables in general parenting. Journal of Child Health Care, 17(2) 204-214. doi: 10.1177/1367493512456107

Alvarenga, P., Weber, L. N. D., \& Bolsoni-Silva, A. T. (2016). Cuidados parentais e desenvolvimento socioemocional na infância e na adolescência: Uma perspectiva analítico-comportamental. Revista Brasileira de Terapia Comportamental e Cognitiva, 18(1), 4-21. Recuperado de http://www.usp.br/rbtcc/ index.php/RBTCC/article/viewFile/827/456

Bardin, I. A. S., Mari, J.J., \& Caeiro, M. F. (2003).Validação da versão brasileira do "Child Behavior Checklist" (CBCL) (Inventário de Comportamentos da Infância e Adolescência): dados preliminares. Revista ABP-APAL, 17(2), 55-66. Recuperado de http:// pesquisa.bvs.br/brasil/resource/pt/psi-1346

Berry, D., \& O’Connor, E. (2010). Behavioral risk, teacher-child relationships, and social skill development across middle childhood: A child-byenvironment analysis of change. Journal of Applied Developmental Psychology, 31(1), 1-14. doi: 10.1016/j. appdev.2009.05.001

Bolsoni-Silva, A. T. Marturano, E. M, \& Loureiro, S. R. (2011). Estudos de confiabilidade e validade do questionário de respostas socialmente habilidosas versão para pais - QRSH-Pais. Psicologia: Reflexão e Crítica, 24(2), 1-9. doi: 10.1590/ S0102-79722011000200003

Bolsoni-Silva, A. T., \& Carrara, K (2010). Habilidades sociais e análise do comportamento: Compatibilidades e dissensões conceitual-metodológica. Psicologia em Revista, 16(2), 330-350. Recuperado de http:// pepsic.bvsalud.org/scielo.php?script=sci_arttext\& pid $=$ S1677-11682010000200007

Bolsoni-Silva, A. T., \& Loureiro S. R. (2011). Práticas educativas parentais e repertório comportamental infantil: Comparando crianças diferenciadas pelo comportamento. Paidéia, 21 (48) 61-71. doi: 10.1590/S0103-863X2011000100008.

Bolsoni-Silva, A. T., \& Marturano, E. M. (2010). Evaluation of Group Intervention for Mothers/ Caretakers of Kindergarten Children with Externalizing Behavioral Problems. Revista Interamericana de Psicologia/Interamerican Journal of Psychology, 44(3), 411-417. Recuperado de http://www.redalyc.org/ comocitar.oa?id $=28420658002$

Bolsoni-Silva, A. T., Loureiro, S. R. (2010). Validação do roteiro de entrevista de Habilidades Sociais Educativas Parentais (RE-HSE-P). Avaliação Psicológica, 9, 63-75, 2010.

Bolsoni-Silva, A. T., Loureiro, S., \& Marturano, E. M. (2016). Roteiro de entrevista de habilidades sociais educativas parentais (RE-HSE-P). Manual Técnico. São Paulo: HOGREFE/Cetepp.

Borden, L. A., Herman, K. C., Stormont, M., Nidhi Goel, N., Darney, D., Reinke, W. M., \& Webster-Stratton, C. (2014). Latent profile analysis of observed parenting behaviors in a clinic sample. Journal Abnormal Child Psychology, 42, 731-742. doi: 10.1007/s10802-013-9815-z

Buehler, C., \& Gerard, J. M. (2013). Cumulative family risk predicts increases in adjustment difficulties across early adolescence. Journal of Youth and Adolescence, 42(6), 905-920. doi: 10.1007/ s10964-012-9806-3

Buyse, E., Verschueren, K., Doumen, S., Van Damme, J., \& Maes, F. (2008). Classroom problem behavior and teacher-child relationships in kindergarten: The moderating role of classroom climate. Journal School Psychology, 46(4), 367-391. doi: 10.1016/j. jsp.2007.06.009

Cohen, J. (1988). Statistical Power Analysis for the Behavioral Sciences. New York, NY: Routledge Academic.

Cosentino-Rocha, L. \& Linhares, M. B. M (2013). Temperamento de crianças e diferenças de gênero. Paidéia, 23(54), 63-72. doi: 10.1590/1982-43272354201308 
Crestani, A. H., Mattana, F., Moraes, A. B., \& Souza, A. P. R. (2013). Socioeconomic, obstetric, demographic and psychosocial factors as risk to child development. Revista CEFAC, 15(4), 847-856. doi: 10.1590/S1516-18462013000400013

Falcão, A. P., \& Bolsoni-Silva, A. T. (2015). Intervention in Social Skills: The Behavior of Children from the Perspective of Parents and Teachers. British Journal of Education, Society \& Behavioural Science, 11(3), 1-18. doi: 10.9734/BJESBS/2015/20033

Freitas, A. P. C. O., \& Piccinini, C. A. (2010). Práticas educativas parentais em relação ao filho único e ao primogênito. Estudos de Psicologia, 27(4), 515-528. doi: 10.1590/S0103-166X2010000400009

Gomide. P. I. C. (2006). Inventário de estilos parentais. Modelo Teórico: Manual de Aplicação, apuração e interpretação. Petrópolis: Vozes.

Gomide. P. I. C. (2006). Inventário de estilos parentais. Modelo teórico: Manual de aplicação, apuração e interpretação. Petrópolis, RJ: Vozes.

Goodman, S. H., Rouse, M. H., Connell, A. M., Broth, M. R., Hall, C. M., \& Heyward D. (2011). Maternal depression and child psychopathology: A meta-analytic review. ClinicalChild Family Psychology Review, 14(1),1-27. doi: 10.1007/s10567-010-0080-1

Hayden, E. P., \& Mash, E. J. (2014). Child psychopathology: A developmental-systems perspectives. Em E. J. Mash \& R. A. Barkley, Child psychopathology (pp. 3-72). New York/London: The Guilford Press.

Hudson, J. L., Flannery-Schroeder, E., \& Kendall, P . C. (2004). Primary prevention of anxiety disorders. Em D. J. A. Dozois \& K. S. Dobson (Eds.). The Prevention of Anxiety and Depression: Theory, Research and Practic, (101-130). Washington, DC: American Psychology Association.

Kettler, R. J., Elliott, S. N., Davies, M., \& Griffin, P. (2011). Testing a multi-stage screening system: Predicting performance on Australia's national achievement test using teachers' ratings of academic and social behaviors. School Psychology International, 33(1), 93111. doi: $0.1177 / 0143034311403036$

Kim, M., Doh, H., Hong, J. S., \& Choi, M. (2011). Social skills training and parent education programs for aggressive preschoolers and their parents in South Korea. Children and Youth Services Review, 33(6), 838845. doi: 10.1016/j.childyouth.2010.12.001
Kroenke, K., Spitzer, R. L., \& Williams, J. B. (2001). The PHQ-9: Validity of a brief depression severity measure. Journal of General Internal Medicine, 16(9), 606-613. doi: 10.1046/ j1525-14972001016009606x

Landale, N. S., Lanza, S. T.; Hillemeier, M., Oropesa, R. S. (2013). Health and development among Mexican. Black and white preschool children: An integrative approach using latent class analysis. Demografic Research, 28(44), 1302-1338. Recuperado de https://www.demographic-research.org/volumes/vol28/44/default.htm

Leman, P. J., \& Bjornberg, M. (2010). Conversation, development, and gender: A study of changes in children's concepts of punishment. Child Development, 81(3), 958-971. doi: 10.1111/j.1467-8624.2010.01445.x

Leme, V. B. R., \& Bolsoni-Silva, A. T. (2010). Habilidades sociais e problemas de comportamento: Um estudo exploratório baseado no modelo construcional. Alethéia, 31, 149-167. Recuperado de http:// pepsic.bvsalud.org/scielo.php?script=sci_ arttext\&pid=S1413-03942010000100013

Leme, V. B. R., \& Marturano, E. M. (2014). Preditores de comportamentos e competência acadêmica de crianças de famílias nucleares, monoparentais e recasadas. Psicologia: Reflexão e Crítica, 27(1), 153-162. doi: 10.1590/S0102-79722014000100017

Lins, T. C. S., Alvarenga, P., Santos, C. P., Almeida, P., \& Santos, H. C. (2012). Problemas externalizantes e agressividade infantil: uma revisão de estudos brasileiros. Arquivos Brasileiros de Psicologia, 64, 57-75. Recuperado de http://pepsic. bvsalud.org/scielo.php?script=sci_arttext\&pi$\mathrm{d}=$ S1809-2672012000300005

Lucas, N., Nicholson, J. M., \& Erbas, B. (2013). Child mental health after parental separation: The impact of resident/non-resident parenting, parent mental health, conflict and socioeconomics. Journal of Family Studies, 19(1), 53-56. Recuperado de https://openresearch-repository.anu.edu.au/bitstream/1885/10543/3/Lucas_ChildMental2013. pdf

Marin, A. H., Piccinini, C. A., Gonçalves, T. R., \& Tudge, J. R.H. (2012). Práticas educativas parentais, problemas de comportamento e competência social de crianças em idade pré-escolar. 
Estudos de Psicologia, 17(1), 5-13. doi: 10.1590/ S1413-294X2012000100002

Martín, V., Granero, R., \& Ezpeleta, L. (2014). Comorbidity of oppositional defiant disorder and anxiety disorders in preschoolers. Psicothema, 26(1), 27-32. doi: $10.7334 /$ psicothema2013.75

Osório, F. L., Mendes, A. V., Crippa, J. A., \& Loureiro, S. R. (2009). Study of the discriminative validity of the PHQ-9 and PHQ-2 in a sample of Brazilian women in the context of primary health care. Perspective Psychiatric Care, 45(3), 216-227. doi: 10.1111/j.1744-6163.2009.00224.x

Patterson, G. R., Reid, J., \& Dishion, T. (1992). Antisocial boys. Santo André, SP: ESETec.

Pizeta F. A., Silva T. B. F., Cartafina M. I. B., \& Loureiro S. R. (2013). Depressão materna e riscos para o comportamento e a saúde mental das crianças: Uma revisão. Estudos de Psicologia (Natal), 18(3), 429437. doi: 10.1590/S1413-294X2013000300003

Price, J. M., Chiapa, A., \& Walsh, N. E. (2013). Predictors of externalizing behavior problems in early elementary-aged children: The role of family and home environments. The Journal of Genetic Psychology: Research and Theory on Human Development, 174(4), 464-471. doi: 10.1080/00221325.2012.690785

Trepat, E., Granero, R., \& Ezpeleta, L. (2014). Parenting practices as mediating variables between parents' psychopathology and oppositional defiant disorder in preschoolers. Psicothema, 26(4), 497-504. doi: $10.7334 /$ psicothema2014.102

Wichstrøm, L., Belsky, J., \& Berg-Nielsen, T. S. (2013). Preschool predictors of childhood anxiety disorders: Aprospective community study. Journal of Child Psychology and Psychiatry, 54(12), 1327-1336. doi: $10.1111 /$ jcpp.12116

Xing, X., \& Wang, M. (2013). Sex differences in the reciprocal relationships between mild and severe corporal punishment and children's internalizing problem behavior in a Chinese sample. Journal of Applied Developmental Psychology, 34, 9-16. doi: 10.1016/j.appdev.2012.09.004

Recebido em: 20/01/2018

Reformulado em: 26/06/2018

Aprovado em: 05/07/2018

Sobre as autoras:

Alessandra Turini Bolsoni-Silva é psicóloga, mestre em Educação Especial e doutora em Psicologia pela Universidade de São Paulo, docente nos Cursos de Psicologia e Pós-Graduação em Psicologia do Desenvolvimento e Aprendizagem na Unesp-Bauru. Trabalha com Análise Aplicada do Comportamento em Psicologia Clínica e em Psicologia do Desenvolvimento em diferentes temáticas quanto a avaliação e intervenção em habilidades sociais.

ORCID: 0000-0001-8091-9583

E-mail: bolsoni@fc.unesp.br

Sonia Regina Loureiro é psicóloga, mestre e doutora em Psicologia Clínica pela Universidade de São Paulo, docente da Faculdade de Medicina de Ribeirão Preto/ USP, orientadora junto aos Programas de Pós-Graduação em Psicologia da FFCLRP-USP e em Saúde Mental da FMRP-USP. Desenvolve atividades de pesquisa com instrumentos e procedimentos de avaliação em diferentes contextos psicossociais.

ORCID: 0000-0001-9423-2897

E-mail: srlourei@fmrp.usp.br

Contato com as autoras:

Faculdade de Ciências - Unesp/Bauru

Av. Eng. Luiz Edmundo Carrijo Coube, 14-01

Bauru-SP, Brasil

CEP: 17033-360

Psico-USF, Bragança Paulista, v. 24, n. 1, p. 69-83, jan./mar. 2019 